\title{
Reaction Kinetics of Photoactive Defects in Semiconductor Dissolution
}

\author{
Eric A. Robertson III and H. Scott Fogler \\ Dept. of Chemical Engineering, University of Michigan, Ann Arbor, MI 48109
}

\begin{abstract}
Uniformity and predictability are the principal qualities sought for all wet chemical etches. The establishment of these qualities, however, is hindered by a number of factors, such as nonuniformities in the starting material and random fluctuations in the local temperature and reactant concentration, which can lead to variations in etch rate across the surface of a wafer. The effects of variations in the local etch rate on the morphological development of an etching surface and on the overall etch rate of the semiconductor are discussed. The system studied was an $\mathrm{Al}_{0.4} \mathrm{Ga}_{0.6} \mathrm{As} / \mathrm{GaAs}$ heterostructure photodissolved in nitric acid using 730-nm laser light. Defects in the Al$\mathrm{GaAs}$ layer, which etched faster than the surrounding material, were responsible for variations in the etch rate. The defects also exhibited a degree of photosensitivity that has not been previously observed. It was also found that not all of the defects spanned the AlGaAs epilayer. A model for the overall etch rate was based on a system of noninteracting cylindrical defects with a distribution in depths.
\end{abstract}

\section{Introduction}

Uniform, or at least predictable, etching of semiconductor layers is necessary for the fabrication of microelectronic and micromechanical devices. However, the semiconductor material always has some imperfections, or defects, associated with it, usually incorporated during material growth, but other processing steps may also introduce defects (Stirland and Straughan, 1976; Mahajan, 1984; Mil'vidskii, 1989). These defects can have etch rates that are significantly different from the rest of the crystal, leading to either etch pits or hillocks depending on whether the defects etch faster or slower than the rest of the crystal. For the purposes of device fabrication, uncontrollable formations are undesirable and are to be minimized. However, an understanding of the etch behavior of defects may lead to opportunities in which the defects can serve a useful purpose.

In this article, a system of random defects in a semiconductor heterostructure is studied to determine how the defects alter the overall etch rate. The defects are represented by a model heterogeneity, present only in the epilayer of the heterostructure, that etches faster than the rest of the epilayer. Comparisons are made to an $\mathrm{AlGaAs} / \mathrm{GaAs}$ heterostructure photoetched in nitric acid. A governing equation is developed to describe the expected behavior of an arbitrary distribution of the model heterogeneities.

\section{Materials and Apparatus}

The photodissolution experiments used a closed-circuit system to monitor the dissolution rate in $250 \mathrm{~mL}$ of $5 \mathrm{wt} \%$ aqueous nitric acid and an expanded laser beam as a monochromatic photon source. The dissolution rates and etch depths were inferred from the oxidation current measured using a potentiostat in the standard three-electrode configuration with an $\mathrm{AlGaAs} / \mathrm{GaAs}$ heterostructure as the working electrode, a platinum counterelectrode, and an SCE reference electrode. The dissolution rates and etch depths were verified by atomic absorption spectroscopy and surface profilometry, respectively. The applied bias was $+0.5 \mathrm{~V}_{\mathrm{SCE}}$ for all experiments, unless otherwise noted. The details of the sample preparation and the system setup are described in detail elsewhere (Robertson and Fogler, 1994).

The heterostructures used were $2-\mu \mathrm{m} n-\mathrm{Al}_{0.4} \mathrm{Ga}_{0.6} \mathrm{As}$ epilayers on an $n$-GaAs substrate. These heterostructure samples were grown by molecular beam epitaxy on a (100) $n$-GaAs substrate with a dopant concentration of $10^{18}$ silicon atoms $/ \mathrm{cm}^{3}$. These materials were chosen because of their suitable optical properties and because of their importance to microelectronic devices.

A laser beam supplied the photons necessary for the generation of holes that lead to the oxidation of arsenic at the surface. The $730-\mathrm{nm}$ light was produced using a dye laser 
with pyridine 2 dye, which is tunable to wavelengths between 700 and $800 \mathrm{~nm}$ with an uncertainty of less than $5 \mathrm{~nm}$. In order to minimize radial variations in etch rate, the laser beam was expanded to several sample diameters, completely covering the surface of the sample with an approximately uniform intensity. The uniformity was established by erecting an aperture slightly larger than the sample and expanding the beam until the aperture could be moved one sample radius without noting a change in the power through the aperture.

The laser-induced rise in the surface temperature of the sample was negligible because of the convective heat transport from the rotating disk and the relatively low intensity of the laser beam (Robertson and Fogler, 1994). The maximum rise in surface temperature is less than $1.2^{\circ} \mathrm{C}$ based on a heat-transfer coefficient (Owen and Rogers, 1989) of 0.7 $\mathrm{W} / \mathrm{cm}^{2} \cdot{ }^{\circ} \mathrm{C}$ and a laser intensity of $0.85 \mathrm{~W} / \mathrm{cm}^{2}$ (the highest used in the experiments).

\section{Heterogeneity Model}

The heterogeneities that lead to variations in etch rate across an exposed surface may be intentionally introduced, for example, damage produced by a focused ion beam for the purpose of forming regions where photoelectrochemical dissolution is suppressed (Khare and $\mathrm{Hu}, 1992$; Cummings et al., 1986). Heterogeneities may also be the result of uncontrollable factors in previous processing steps, for example, dislocations formed during growth. The number of ways the heterogeneities could be arranged is unlimited, with variations occurring in the number, positions, sizes, or even the etch rates of the heterogeneities themselves. The first step in studying the effects of heterogeneities is to formulate the geometry of a model heterogeneity. The second step is then to assign a dissolution behavior to the different regions of the heterogeneity and evaluate how a single defect dissolves. A system of defects is then constructed and analyzed based on the cumulative behavior of the individual defects.

\section{Geometry of the defects}

The initiation of a defect is in general a random event. For the purposes of this article, a heterogeneity, or defect, is defined as a circular region with a constant radius initiated during the growth of a semiconductor epilayer. The defect propagates upward as the epilayer grows (see Figure 1). Thus the radius and depth of each defect as well as the number of defects are random variables. By assuming a constant radius reflecting the average radius of the defects, the complexity of the model is reduced. Such a stipulation is reasonable given the constraints already imposed by the cylindrical geometry. The number of defects is chosen at random from a given range with equal probability, the so-called uniform distribution.

The depths are sampled at random from a uniform distribution, which is a limiting form of the Poisson distribution. This choice is based on the assumption that a random event initiates a defect between time $t$ and $t+\Delta t$ with a probability proportional to $\Delta t$. This is equivalent to assuming that the random event occurs between a distance $z$ and $z+\Delta z$ from the substrate, with a probability proportional to $\Delta z$. The proportionality constant is defined as $1 / \mu$. The thickness of the epilayer grown before a defect is initiated, $z$, is Poisson distributed. Therefore the expected value of $z$ is $\mu$. The distri-

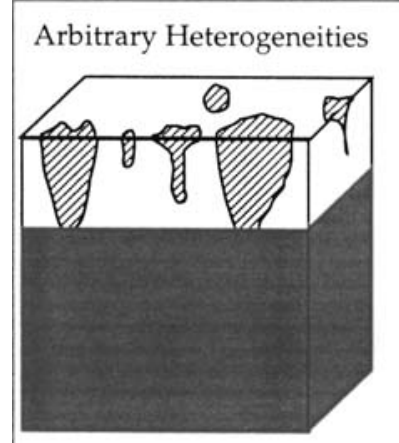

A

non-defect $\square$ defect $\square$ substrate

Figure 1. (a) System of arbitrary heterogeneities and (b) system of model heterogeneities.

The epilayer thickness, $T$, and the defect depth, $d_{i}$, are denoted in (b).

bution is renormalized to guarantee at least one defect-initiating event in the epilayer, that is, between $z=0$ and $z=T$, where $T$ is the thickness of the epilayer. Renormalization is necessary because the number of defects is generated prior to the assignment of depths. The distribution of defect depths, $f_{d}$, is obtained by a simple change of variable, $d=T-z$. Consequently, $T-\mu$ is the mean defect depth. It should be noted that $f_{z}$ is the distribution function for sampling from the Poisson distribution before renormalization and rejecting any $z$ greater than $T$ :

$$
f_{z}=\frac{1}{\mu} \frac{e^{-z / \mu}}{\left(1-e^{-T / \mu}\right)} \rightarrow f_{d}=\frac{e^{d / \mu}}{\mu\left(e^{T / \mu}-1\right)}
$$

When $\mu / T>10$, the defect depths are approximately uniformly distributed between 0 and $T$. An estimate for $\mu / T$ is obtained from the fraction of the surface initially covered by defects, $\epsilon$, at the surface of the sample, that is, $\mu=-T / \ln (1$ $-\epsilon)$. For $\epsilon$ less than 0.02 -as indicated by the samples studied-then $\mu / T$ is greater than 50 . Therefore the defect depths are treated as uniformly distributed.

\section{Stages of defect dissolution}

It is instructive to consider the dynamics of a single defect before trying to describe the action of many defects dissolving simultaneously. The area immediately surrounding a defect will, in general, undergo three stages in the course of its dissolution (see Figure 2): dissolution of the defect (Figure 2a), dissolution of the remaining epilayer down to the substrate (Figure 2b), and the dissolution of the substrate itself (Figure 2c). Clearly, it is the evolution of the geometry of the defect that governs the changes in etch rate over time. For each stage, the total current, $J(t)$, can be calculated knowing the geometry of the defect at that particular time and the local etch rates for the different regions. The amount of material removed-or equivalently, the charge transferred, $Q(t)$ - is given by simple integration of the current over time. 


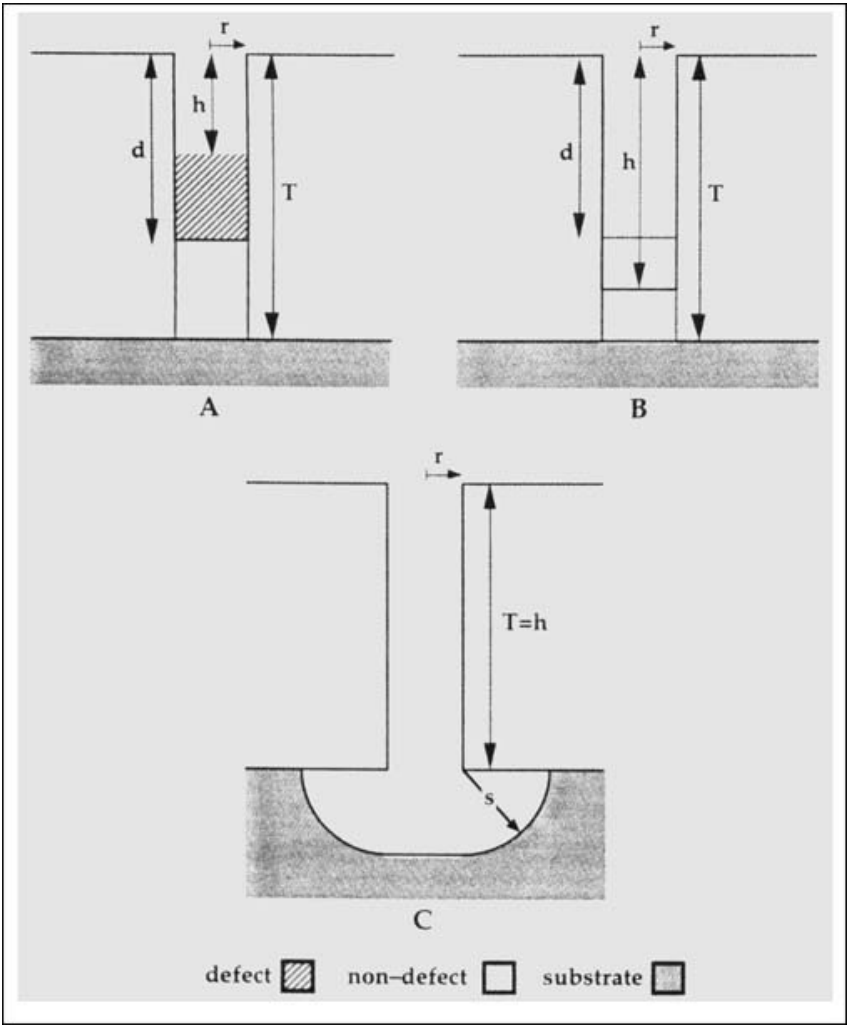

Figure 2. Three stages involved in the dissolution of an individual defect: (a) Stage I, $0 \leq t<t_{1}$; (b) Stage II, $t_{1} \leq t<t_{11}$, and (c) Stage III, $t_{11} \leq t ; S$ is defined as $\Phi j_{s}\left(t-t_{\| \mid}\right)$.

Stage I (Figure 2a) is the dissolution of the defect itself, forming a channel into which the etchant flows. The depth of the channel, denoted as $h$, increases linearly with time because the etch rate is reaction-rate limited (Robertson and Fogler, 1994). The current is due to the dissolution of the sidewall and bottom of the channel. The height, current and charge are given by Eqs. 2, 3 and 4, respectively. The dissolution rates are treated in terms of current densities $\left(\mathrm{A} / \mathrm{cm}^{2}\right)$ rather than the equivalent mole fluxes $\left(\mathrm{mol} / \mathrm{cm}^{2} \cdot \mathrm{s}\right)$ because of the electrochemical nature of the dissolution process. Therefore, the equations of the depth of the etch channel, $h$, the current density, $J$, and the charge, $Q$, are

$$
\begin{gathered}
h(t)=\frac{M w}{\rho n F} j_{d} t \equiv \Phi j_{d} t \\
J_{\mathrm{I}}(t)=\pi r^{2} j_{d}+2 \pi r h(t) j_{n} \\
Q_{\mathrm{I}}(t) \equiv \int_{0}^{t} J_{\mathrm{I}}(\tau) d \tau=\pi r^{2} j_{d} t\left(1+\frac{\Phi j_{n} t}{r}\right),
\end{gathered}
$$

where $\Phi$ is the charge-specific volume of the layer $\left(\mathrm{cm}^{3} / C\right) ; \rho$ is the mass density $\left(\mathrm{g} / \mathrm{cm}^{3}\right) ; M w$ is the formula weight; $n$ is the number of equivalents per gmol $\left(n=6\right.$ for $\mathrm{Al}_{0.4} \mathrm{Ga}_{0.6} \mathrm{As}$ and $\mathrm{GaAs}) ; \mathbf{F}$ is Faraday's constant $\left(9.63 \times 10^{4} \mathrm{C} / \mathrm{eq}\right)$; and $j_{d}$ and $j_{n}$ are the defect and nondefect rates. The nondefect rate is assumed to be much less than the defect rate, that is, $j_{n} \ll j_{d}$, because pits are observed experimentally rather than hillocks. Therefore, broadening of the channel is negligible. Equations 2-4 are valid until the defect is exhausted at time $t_{\mathrm{I}} \equiv d / \Phi j_{d}$, where $d$ is the depth of the defect. The minimum time required to etch through the epilayer occurs when the defect spans the epilayer $(d=T)$ and is denoted by $\alpha$.

At $t=t_{\mathrm{I}}$, the etch rate slows, marking the transition into Stage II (Figure $2 \mathrm{~b}$ ) where the channel continues progressing toward the substrate, but at the slower rate of $\Phi j_{n}$. The etch channel depth, current, and charge during Stage II are given by Eqs. 5-7. These equations are valid until the substrate becomes exposed to the etchant, that is, $h(t)=T$, at time $t_{\mathrm{II}}$. Time $t_{\mathrm{II}}$ is given mathematically as $t_{\mathrm{II}} \equiv t_{\mathrm{I}}+\left[(T-d) / \Phi j_{n}\right]$.

$$
\begin{gathered}
h(t)=d+\Phi j_{n}\left(t-t_{\mathrm{I}}\right) \\
J_{\mathrm{II}}(t)=\pi r^{2} j_{n}+2 \pi r h(t) j_{n} \\
Q_{\mathrm{II}}(t)=Q_{\mathrm{I}}\left(t_{\mathrm{I}}\right)+\pi r^{2} j_{n}\left(t-t_{\mathrm{I}}\right)\left(1+\frac{2 d+\Phi j_{n}\left(t-t_{\mathrm{I}}\right)}{r}\right) .
\end{gathered}
$$

If $r / T \gg 1$, then broadening of the channel is negligible during Stage II, when $j_{d}$ is effectively zero. For example, if $r / T$ $=15$, the maximum error is approximately $8 \%$ for $d=0$, and it decreases with deeper defects.

At $t=t_{\mathrm{II}}$, the substrate is exposed and the dissolution enters its final stage, Stage III (Figure 2c). The exposed sidewall area is now $2 \pi r T$ and remains constant from this point on. The substrate is assumed to be uniform, with a constant, isotropic etch rate, $j_{s}$, greater than $j_{n}$. The channel depth, current, and charge for $t \geq t_{I I}$ are

$$
\begin{gathered}
h(t)=T \\
J_{\mathrm{II}}=2 \pi r T j_{n}+A\left(t-t_{\mathrm{II}}\right) j_{s} \\
Q_{\mathrm{III}}=Q_{\mathrm{II}}\left(t_{\mathrm{II}}\right)+2 \pi r T j_{n}\left(t-t_{\mathrm{II}}\right)+\Phi V\left(t-t_{\mathrm{II}}\right),
\end{gathered}
$$

where $A(\zeta)$ and $V(\zeta)$ are the area and volume of the pit. The $A(\zeta)$ and $V(\zeta)$ increase over time according to the following expressions (Hubbard and Antonsson, 1994):

$$
\begin{array}{r}
A\left(t-t_{\mathrm{II}}\right)=\pi r^{2}+\pi^{2} r\left[\Phi j_{s}\left(t-t_{\mathrm{II}}\right)\right]+2 \pi\left[\Phi j_{s}\left(t-t_{\mathrm{II}}\right)\right]^{2} \\
V\left(t-t_{\mathrm{II}}\right)=\pi r^{2}\left[\Phi j_{s}\left(t-t_{\mathrm{II}}\right)\right]+\frac{\pi^{2} r}{2}\left[\Phi j_{s}\left(t-t_{\mathrm{II}}\right)\right]^{2} \\
+\frac{2 \pi}{3}\left[\Phi j_{s}\left(t-t_{\mathrm{II}}\right)\right]^{3}
\end{array}
$$

With the action of a single defect defined, the effect of many defects can be calculated by summing the contributions of individual defects.

\section{Calculation of the average current density}

The action of many defects dissolving simultaneously can be substantially different than the action of a single defect. The best characterization of a system containing many defects is the rate of mass removal (current) as a function of the total mass removed (volume), normalized by the initial surface area for comparative purposes. The current is simply the current for the nondefect region plus the sum of the currents 
for each defect. Because the depth of individual defects varies, the sum of the currents for each defect is not equal to the contribution for one defect times the total number of defects.

By definition, once a defect is initiated, it does not terminate and must emerge at the surface of the sample. Thus the total cross-sectional area of the defects increases as the distance from the substrate increases. Because the sample etches from the surface back toward the substrate, the contribution of the defects to the total current will decrease over time as the shallower defects are exhausted. This trend continues until the substrate is first exposed. At this point, current contributions from the substrate increase as existing pits grow in size and as new pits are formed when the shallower defects eventually etch through to the substrate.

As the various pits grow, they will begin to coalesce, and the total area and volume are no longer simply the sum of the area and volume of each pit. The time it takes for the pits to coalesce obviously depends on the distances between pit centers, the initial radius of the etch pits, and $j_{s}$. The coalescence of the pits could be incorporated knowing the locations of the pit centers and adjusting the area and volume from Eqs. 11 and 12 for the interactions. However, there are only a few pits evolving over a short period of time, and thus the coalescence of pits is explicitly neglected in this application. Coalescence will be addressed in a future publication.

Systems of noninteracting defects are simulated by first generating a sequence of defects with randomly selected depths. Equations 2-7 are then evaluated for each defect at a prescribed set of times. The current and charge contributions of each individual defect are summed and added to the contribution from the nondefect surface, thus supplying the total current and charge for the system. The total current and charge are subsequently normalized by the initial surface area to give an average current density and an average etch depth.

The result of a typical realization using 25 defects is shown in Figure 3, where the average current density, $\bar{J}(t)$, is plotted as a function of the average etch depth, $\bar{D}(t)$. The particular parameters used are listed with the figure. There are three stages in the dissolution of the entire surface (marked in Figure 3) that correspond roughly to the three stages described earlier for the dissolution of a single defect. In the first stage (I in Figure 3), the current decreases as the defects are exhausted. The discrete jumps are due to the finite number of defects; a larger number of defects would show a more continuous transition. In the second stage (II in Figure 3), the current reaches a minimum when all of the defects are exhausted. This minimum may last for an extended period of time if there are no layer-spanning defects. The duration of the minimum will decrease with deeper defects. Finally, in Stage III, the substrate is exposed and the current rises because the substrate dissolution rate is much greater than the nondefect dissolution rate, that is, $j_{s}>j_{n}$, and because the pits grow in size as the etch progresses. The discrete jumps in current in Stage III are also due to the finite number of defects, reflecting the time delay in forming pits associated with shallower defects. Also shown on Figure 3 is the average of many different realizations from the same depth distribution. The gray lines represent the envelope for realizations falling within one standard deviation of the average, thus providing an indication of what may be expected in an actual dissolu-

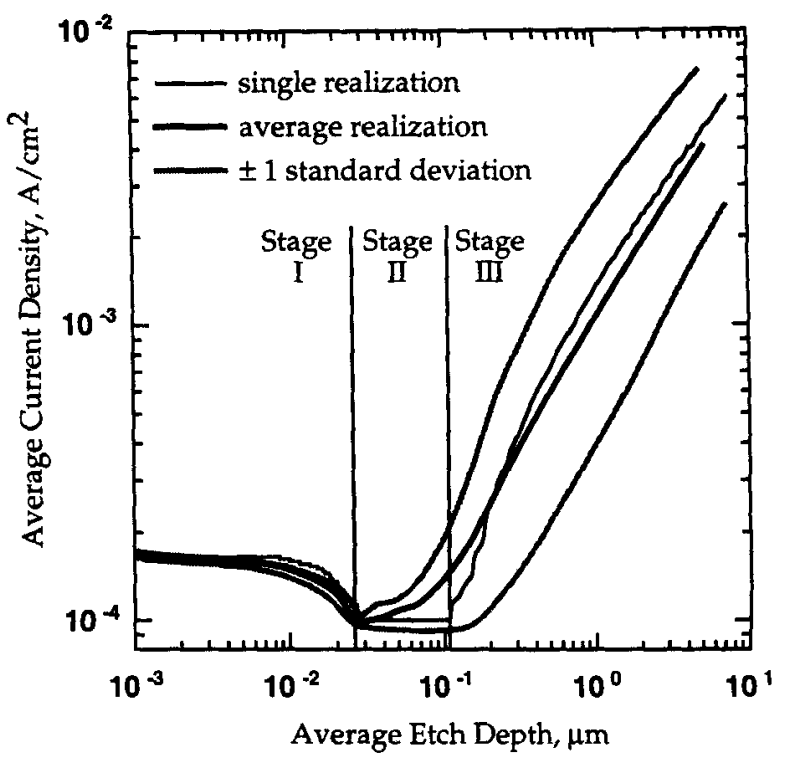

Figure 3. Typical realization using 25 defects generated at random.

The model parameters used were $j_{n}=0.001 \mathrm{~A} / \mathrm{cm}^{2}, j_{d}=0.01$ $\mathrm{A} / \mathrm{cm}^{2}, j_{s}=0.05 \mathrm{~A} / \mathrm{cm}^{2}, r=0.003 \mathrm{~cm}, T=0.0002 \mathrm{~cm}$, and $A_{o}=0.1 \mathrm{~cm}^{2}$.

tion experiment. It is important to note that the variance is a result of sampling defect depths from a uniform distribution. Therefore the variance will depend on the number of defects contained on the sample.

\section{Experimental Etching Results}

The model heterogeneity, as described previously, is based on observations from photoetching $\mathrm{Al}_{0.4} \mathrm{Ga}_{0.6}$ As epilayers in $\mathrm{HNO}_{3}$ under closed-circuit conditions. These observations include the morphology of the epilayer at the completion of the experiments and the dissolution rate, or current, as a function of time. The surface morphology forms the basis of the geometry of the model heterogeneities and the pits formed in the substrate, in particular that the defects are circular and have varying depths. The currents provide estimates for the dissolution rates used in the defect model. The cause of the defects is unknown, but it is thought to be a combination of defect photosensitivity and bias. Similar results were obtained using a variety of samples from different wafers; therefore the effect is not limited to a single anomalous wafer.

\section{AlGaAs as a model system}

An AlGaAs/GaAs heterostructure photoetched in $5 \mathrm{wt} \%$ $\mathrm{HNO}_{3}$ using 730-nm laser light and an overpotential of +0.5 $\mathrm{V}_{\mathrm{SCE}}$ met the three requirements of the model heterogeneities stated earlier, with a few minor exceptions as noted below. The etch results using various intensities, including two runs without illumination, are shown in Figure 4.

The first assumption in the heterogeneity model is that the defects are cylindrical regions with an etch rate, $j_{d}$, which is greater than that of the nondefect regions, $j_{n}$. Figure 5 is a SEM of the AIGaAs epilayer showing the result of a typical defect observed after etching. The circular hole on the left is 


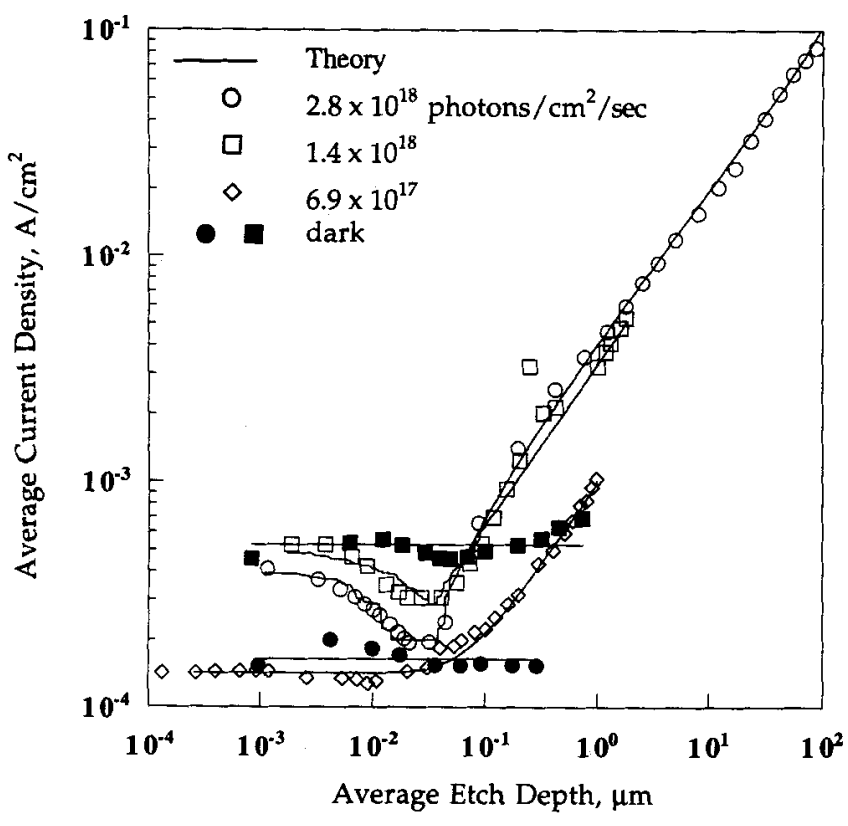

Figure 4. Dissolution experiments using 730-nm laser at several different intensities.

The equivalent photon currents used were (1) 0.45 (E), (2) 0.22 (G), (3) $0.11(\mathrm{~A})$, (4) $0.00(\mathrm{~J})$, and (5) 0.00 (B) $\mathrm{A} / \mathrm{cm}^{2}$. Model results are represented by the solid lines. The epilayer thickness is $2 \mu \mathrm{m}$.

the hole remaining after the defect was dissolved. The SEM also clearly shows the degree of undercutting in the GaAs substrate resulting from the transparency of the AlGaAs epilayer to the $730-\mathrm{nm}$ light. The diameters ranged from 20 to $80 \mu \mathrm{m}$, with the average being approximately $60 \mu \mathrm{m}$. Other micrographs also showed that not all of the defects reached the substrate during the course of the experiment, implying a distribution in defect depths. This sample justifies the use of the cylindrical geometry in the heterogeneity model. Further-

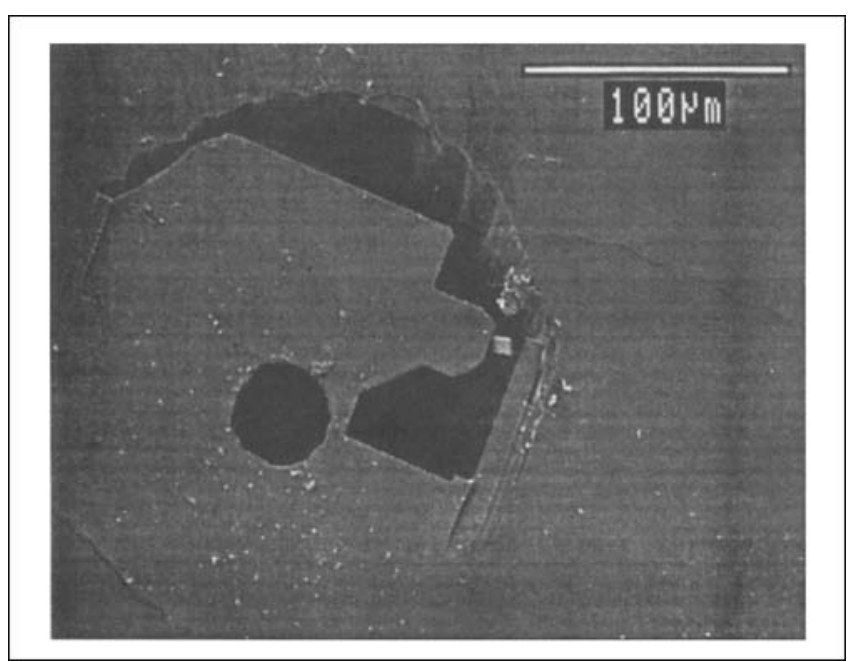

Figure 5. Scanning electron micrograph depicting the circular nature of the defects observed in the $\mathrm{Al}_{0.4} \mathrm{Ga}_{0.6}$ As layer.

Also shown is the degree of undercutting resulting from the transparency of the $\mathrm{Al}_{0.4} \mathrm{Ga}_{0.6}$ As epilayer. more, the minimums observed in Runs $1-3$ of Figure 4 suggest that $j_{d}$ is greater than $j_{n}$, and that the area etching at a rate $j_{n}$ decreases as the etch progresses, a direct result of defect propagation without healing.

The second assumption is that the substrate etches at a constant, isotropic rate, $j_{s}$, which is greater than $j_{n}$. The rate will remain constant as long as the dissolution remains reaction-rate limited. Instead of etching isotropically, GaAs can etch crystallographically, producing hexagonal etch pits (Stirland and Straughan, 1976; Stirland et al., 1986; and Iwaski et al., 1990). The nature of the etch pits was determined by first removing the remaining $\mathrm{AlGaAs}$ layer using a 4-min iodine etch (Malag et al., 1993; and Levine et al., 1983). About half of the major pits in the GaAs substrate appeared to etch crystallographically, while the other half appeared to etch isotropically. In either event, the pits were nearly circular, so the etch was considered to proceed isotropically. Finally, $j_{s}$ is greater than $j_{n}$ because light at a wavelength of $730 \mathrm{~nm}$ substantially accelerates the dissolution of GaAs over the dark rate at the applied bias of $+0.5 \mathrm{~V}_{\mathrm{SCE}}$, but does not substantially accelerate the dissolution of $\mathrm{Al}_{0.4} \mathrm{Ga}_{0.6}$ As (Robertson and Fogler, 1994; and Khare and Hu, 1993). Also, because the AlGaAs is transparent to the light, the anisotropic etch normally expected with an absorbing mask is not present here, allowing the GaAs substrate to continue to etch under the AlGaAs layer once the GaAs is exposed to the etchant.

The final assumption for the model heterogeneities is that the duration of the etch and the locations of the defects are such that there is no interaction between defects or the pits that eventually form. Pit interaction is nearly inevitable at longer durations regardless of the defect density, so the analysis is not strictly valid at long durations. At short durations, pit interaction may occur if two or more defects were close together. Considerable pit interaction was evident in Run 1, where approximately $50 \%$ of the AlGaAs epilayer had been removed. The durations of the remaining runs were significantly shorter, thus minimizing interactions. For Run 3, only two of the 15 major pits coalesced during the experiment (see Figure 6).

\section{Application of model}

The model was applied to the data shown in Figure 4, using a collection of defects with a distribution of depths determined by sampling a uniform distribution. For the purpose of clarity, only those points necessary to show the trend and the degree of fit are presented in Figure 4; many more points were used in the regression analysis $(53,71$, and 141 for Runs 1,2 , and 3 , respectively). The procedure was to first generate a system of defects, or realization, and then regress the predicted current against the observed current, varying the defect radius, $r$, and the etch rates $j_{n}, j_{d}$, and $j_{s}$ to minimize the error. A weighted least-squares technique and a simplex minimization procedure were used to give the best fit between the realization and the data. It was found that the particular realization had as great an effect on the regression as did the radius or the etch rates; therefore, the regression procedure was repeatedly applied to many different realizations. This process was repeated for Runs 1, 2, and 3 in Figure 4.

The estimated substrate rate compared favorably to the dissolution rates observed for GaAs substrate material re- 


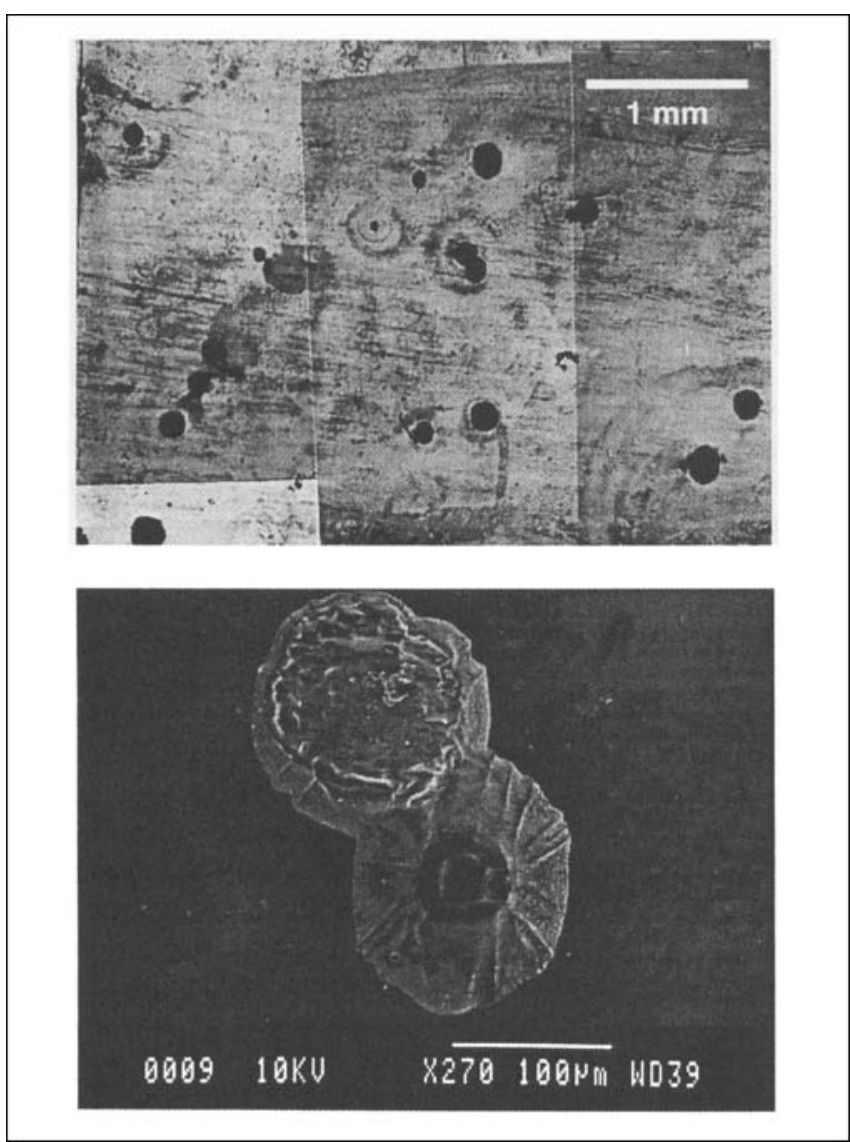

Figure 6. (a) Composite constructed of several optical images of the surface of the sample used in Run 3 of Figure 4 after the remaining AIGaAs layer was removed with a 4-min iodine etch (the scale represents $1 \mathrm{~mm}$ ); (b) SEM of the two coalesced pits in (a).

ported previously in the literature (Robertson and Fogler, 1994). Also, the rates estimated for the nondefect AlGaAs agreed with rates reported in the literature for nitric acid (Robertson and Fogler, 1994; and Fink and Osgood, 1993) and citric acid/peroxide etches (Mao et al., 1994). There are no published rates for the dissolution of defects. Table 1 summarizes the model parameters.

\section{Photoactivity of the defects}

We now need to address how the various rates depend on the light intensity. The intensity dependence of the nondefect rate of $\mathrm{Al}_{0.4} \mathrm{Ga}_{0.6} \mathrm{As}, j_{n}$, is expected to be low because the

Table 1. Model Parameters Estimated for Results in Figure 4

\begin{tabular}{cccccc}
\hline $\begin{array}{c}\text { Run } \\
\text { No. }\end{array}$ & $\begin{array}{c}\text { Defect } \\
\text { Density } \\
\text { No. } / \mathrm{cm}^{2}\end{array}$ & $\begin{array}{c}j_{s} \\
\mathrm{~A} / \mathrm{cm}^{2}\end{array}$ & $\begin{array}{c}j_{d} \\
\mathrm{~A} / \mathrm{cm}^{2}\end{array}$ & $\begin{array}{c}j_{n} \\
\mathrm{~A} / \mathrm{cm}^{2}\end{array}$ & $\begin{array}{c}\text { Radius } \\
\mu \mathrm{m}\end{array}$ \\
\hline 1 & 238 & 0.18 & $4.05 E-02$ & $2.00 E-04$ & 26.2 \\
2 & 438 & 0.10 & $2.33 E-02$ & $2.65 E-04$ & 26.0 \\
3 & 342 & 0.064 & $8.62 E-03$ & $1.26 E-04$ & 11.6 \\
4 & - & - & $1.64 E-04$ & $1.64 E-04$ & - \\
5 & - & - & $5.26 E-04$ & $5.26 E-04$ & - \\
\hline
\end{tabular}

wavelength used was sufficiently beyond the $653 \mathrm{~nm}$ cutoff for $\mathrm{Al}_{0.4} \mathrm{Ga}_{0.6} \mathrm{As}$ (Khare and $\mathrm{Hu}, 1993$ ). The GaAs dissolution rate, $j_{s}$, has been studied under a wide range of intensities and wavelengths, including those used in these experiments (Robertson and Fogler, 1994; Kuhn-Kuhnenfeld, 1978; Adachi and Oe, 1984; Badawy et al., 1990; and Ruberto et al., 1991). Those results are available in the literature and will not be repeated here, except to note that in general the dissolution rate increases with increasing intensity, though not always linearly. It is assumed that the presence of a transparent mask does not alter the etch rate compared to the maskless situation. This may not be true if the mask is not entirely transmitting or if there are diffusion limitations.

The effect of the laser intensity on $j_{d}$, however, is not clear. The model results suggest that $j_{d}$ depends on the intensity of the illumination; however, the currents for Runs 4 and 5, where the intensity was zero, lie between those for Runs 1-3. If $j_{d}$ was independent of the intensity, then an experiment at zero intensity would show trends similar to those with nonzero intensity. However, if $j_{d}$ was strongly influenced by the intensity, presumably higher with higher intensities, then the trend would be for the current to remain constant for a longer period of time. If $j_{d}=j_{n}$, the current would remain constant until the layer was removed; if $j_{d}<j_{n}$, then hillocks would form. The trend in Runs 4 and 5 , where the laser intensity was zero, showed a nearly constant current far beyond the point where the epilayer was etched through in Runs 1-3, thus supporting the latter case of an intensity-dependent heterogeneity.

Though there are other explanations for a constant current as observed in Runs 4 and 5, these explanations are unlikely given the experimental observations. First, a constant current would be produced if there were no defects present in the samples used for Runs 4 and 5. This explanation is unlikely because 15 major etch pits were observed in Run 3 and because defect densities on the order of $300 \mathrm{~cm}^{-2}$ were suggested by the model parameters. Second, instead of $j_{d}$ decreasing with lower light intensities, $j_{n}$ may have increased to a value near $j_{d}$. However, $j_{n}$ tends to decrease with laser intensity and then only slightly (Robertson and Fogler, 1994; and Khare and Hu, 1992). Even the variation in current density observed between Runs 4 and 5 would not indicate a rate nearly equal to the $j_{d}$ estimated for Runs 1-3. Finally, the only other explanation is that there were a large number of deep defects and the etch was terminated before the substrate was exposed. A large number of deep defects would leave pits in the surface of the epilayer; no such pits were observed either under optical or scanning electron microscopy (SEM). (However, a few hillocks were observed, which is consistent with $j_{d}<j_{n}$.)

Therefore it is believed that $j_{d}$ is dependent on the intensity of the illumination.

\section{Expected Behavior of Model Heterogeneities}

The correspondence of the model heterogeneities to the observations is encouraging; but there is still no way to predict in general how a particular sample will behave without an exact description of the defects for that particular sample. However, the expected average current density and average etch depth, $\langle\bar{J}(t)\rangle$ and $\langle\bar{D}(t)\rangle$, can be calculated and then 
used to predict the behavior of an average sample. Similarly, as the sample size increases, the variance decreases, making $\langle\bar{J}(t)\rangle$ and $\langle\bar{D}(t)\rangle$ a better reflection of what might be observed. Most importantly, though, this calculation allows the formulation of rules that govern the behavior of the model heterogeneities, one of which was stated earlier: $\bar{J}(t)$ cannot increase until the substrate is exposed because the defects always propagate upward.

One approach to computing $\langle\bar{J}(t)\rangle$ would be to write Eqs. 3,6 , and 9 for each of the defects and calculate $\langle\bar{J}(t)\rangle$ and $\langle\bar{D}(t)\rangle$ using the definition of an expectation value. However an approach that is mathematically equivalent, but more physically insightful, is to first realize that the variance in $\bar{J}(t)$ decreases as the sample size increases. So for an infinitely large sample, $\bar{J}(t)=\langle\bar{J}(t)\rangle$ and $\bar{D}(t)=\langle\bar{D}(t)\rangle$. Because the model heterogeneities have a simple geometry, the current contributions from different surfaces are well defined. All that remains is to calculate the current contributions from the four types of surfaces exposed to the etchant: (1) the nondefect surface; (2) the ends of the channels; (3) the walls of the channels, and (4) the substrate after exposure to the etchant. Each contribution will be considered separately and then added together and divided by the initial surface area, $A_{o}$, to provide the average current density. Another basis for the average could be used, such as the true area exposed to the etchant, but only the initial surface area can be determined accurately for an experiment.

The current contribution from etching the nondefect portion of the surface is simply

$$
J_{\text {surface }}(t)=(1-\epsilon) A_{o} j_{n},
$$

where $\epsilon$ is the fraction of the surface covered by defects. Equation 13, and all subsequent equations, are valid only as long as there is some epilayer remaining, that is, $t \leq T / \Phi j_{n}$.

The second current contribution, $J_{\text {end }}$, comes from etching the ends of the cylinders. $J_{\text {end }}$ decreases over time as the defects become exhausted. The fraction of defects not exhausted, $Y(t)$, is calculated by integrating $f_{z}$ from the substrate $(z=0)$ to the bottom of the deepest channel $(z=T-$ $\left.\Phi j_{d} t\right) . f_{z}$ was discussed previously, in the section defining the geometry of a defect. Making a change of variable to normalize $z, Y(t)$ becomes

$$
Y(t)=\int_{0}^{1-(t / \alpha)} f_{x} d x
$$

where $x$ is defined as $z / T, \alpha$ is $T / \Phi j_{d}$ (the time required to remove a layer-spanning defect), and $f_{x}$ is the probability density function for $x$ ( $x=0$ corresponds to a defect initiated at the substrate and $x=1$ to a defect initiated at the surface). By definition, $f_{x}$ is zero for $x<0$ and for $x>1$. Using this definition of $Y(t), J_{\text {end }}$ becomes

$$
\begin{aligned}
J_{\mathrm{end}}(t)=\epsilon A_{o} j_{d} Y(t)+\epsilon A_{o} j_{n}[1-Y(t)] & \\
& -\epsilon A_{o} j_{n} \int_{0}^{1} H\left[t-t_{\mathrm{II}}(x)\right] f_{x} d x \\
t_{\mathrm{II}}(x) & =\frac{T-z}{\Phi j_{d}}+\frac{z}{\Phi j_{n}},
\end{aligned}
$$

where $H(\zeta)$ is the Heaviside function and $t_{\mathrm{II}}(x)$ is the time at which a pit begins to form. The first and second terms on the righthand side arise from the active and exhausted defects, respectively. The third term accounts for the loss of end regions when the substrate is exposed at $t=t_{\mathrm{II}}(x)$. The correction is of course zero for $t<t_{\mathrm{II}}(x)$.

The third contribution to $\langle\bar{J}(t)\rangle$ is due to the dissolution of the sidewall, $J_{\text {wall }}$. This contribution increases as the etch progresses because more sidewall area becomes exposed as the channel deepens. However $J_{\text {wall }}$ never goes above $2 \epsilon A_{o} j_{n}$ $(T / r)$, which is when all of the cylinders have reached the substrate. $J_{\text {wall }}$ is negligible compared to the contributions from $J_{\text {surface }}$ and $J_{\text {end }}$ (less than $4 \%$ for $T<r$ and $\epsilon \leq 0.02$ ).

The fourth and final current contribution is the dissolution of the substrate where the defects etch through the epilayer and form pits. The current contribution from an individual pit is $A\left[t-t_{\mathrm{II}}(x)\right] j_{s}$, where $A\left[t-t_{\mathrm{II}}(x)\right]$ is given by Eq. 11 . As a reminder that $A(\zeta<0)$ is not defined, the Heaviside function is included where necessary in the following equations:

$$
J_{\mathrm{pit}}(t)=\frac{\epsilon A_{o} j_{s}}{\pi r^{2}} \int_{0}^{1} H\left[t-t_{\mathrm{II}}(x)\right] A\left[t-t_{\mathrm{II}}(x)\right] f_{x} d x
$$

Since $H(\zeta<0)=0$, the integrals in Eqs. 15 and 17 can be simplified with respect to their limits of integration. First define $\left(j_{d} / j_{n}\right)$ as $\beta+1$ and express $t_{\mathrm{II}}(x)$ in terms of $\alpha$ and $\beta$.

$$
t_{\mathrm{II}}(x)=\alpha(1+\beta x) .
$$

$H(\zeta)=1$ when $[t-\alpha(1+\beta x)] \geq 0$ or, equivalently, when $x$ $\leq((t-\alpha) / \alpha \beta)$. Therefore, the integration need only be carried out between $x=0$ and $x=((t-\alpha) / \alpha \beta)$.

The expected value of the current, $\langle\bar{J}(t)\rangle$, is the sum of these contributions divided by the initial surface area, $A_{o}$ :

$$
\begin{aligned}
\langle\bar{J}(t)\rangle=j_{n}+ & \epsilon\left(j_{d}-j_{n}\right) Y(t)-\epsilon j_{n} \int_{0}^{(t-\alpha) / \alpha \beta} f_{x} d x \\
& +\frac{\epsilon j_{s}}{\pi r^{2}} \int_{0}^{(t-\alpha) / \alpha \beta} A[t-\alpha(1+\beta x)] f_{x} d x .
\end{aligned}
$$

If $j_{x} \gg j_{n}$, then the current contribution from the newly formed pit dominates the correction applied to $j_{\text {end }}$. Thus the third term of Eq. 19 is negligible for $t>\alpha$. For $t<\alpha$, this term is identically zero. Therefore it can always be neglected if $j_{s} \gg j_{n}$.

$\langle\bar{D}(t)\rangle$ is proportional to the time integral of $\langle\bar{J}(t)\rangle$ and is calculated by a simple integration of Eq. 19 with respect to time. The time integral of the last term of Eq. 19 varies as the total volume of all the pits; the volume of a single pit, $V(\zeta)$, is given in Eq. 12:

$$
\begin{array}{r}
\langle\bar{D}(t)\rangle=\Phi\left(j_{n} t+\epsilon\left(j_{d}-j_{n}\right) \int_{0}^{t} Y(\tau) d \tau\right. \\
\left.-\epsilon j_{n} \int_{0}^{t} \int_{0}^{(\tau-\alpha) / \alpha \beta} f_{x} d x d \tau\right) \\
+\frac{\epsilon}{\pi r^{2}} \int_{0}^{(t-\alpha) / \alpha \beta} V[t-\alpha(1+\beta x)] f_{x} d x .
\end{array}
$$


Originally, it was stated that both the number of defects and their individual depths were random events, but the derivations of $\langle\bar{J}(t)\rangle$ and $\langle\bar{D}(t)\rangle$ did not consider any variation in $\epsilon$. Clearly, $\langle\bar{J}(t)\rangle$ will depend linearly on $\epsilon$; therefore, $\epsilon$ is the mean of the distribution used to describe the defect coverage. Of course for an infinite sample, there can be no $\epsilon$ distribution, or more precisely the variance of the distribution goes to zero. Thus, the expected result of etching a sample with the model heterogeneity can be calculated once the distribution of depths is specified. The following section uses these relations with instructive depth distributions to formulate rules about the behavior of the heterogeneities under extreme conditions.

\section{Distributions of defect depths}

The model as applied to the experimental results in Figure 4 used a uniform distribution of defect depths, which was selected on the assumption that defects in the semiconductor epilayers are rare events. However, other distributions could be assumed to reflect the conditions under which the defects were introduced. For example, defects introduced by ion bombardment after growing the epilayer will tend to be shallower rather than deeper. Any unimodal probability distribution function describing the distribution of defect depths will have two extreme cases: one a uniform distribution, and one in which all defects have the same depth. These two cases are discussed separately below, along with the Poisson distribution discussed earlier.

Layer Spanning Defects. If all of the defects are initiated quickly - for example, dislocations in the epilayer that begin as a result of an emergent dislocation in the substrate - then all of the defects will span the epilayer (i.e., $d=T$ ). Under this condition, $\langle\bar{J}(t)\rangle$ and $\langle\bar{D}(t)\rangle$ can be retrieved analytically from Eqs. 19 and 20 using $f_{x}=\delta(x)$, where $\delta(x)$ is the Dirac delta function and $A(\zeta)$ and $V(\zeta)$ are given by Eqs. 11 and 12:

$t<\alpha:$

$$
\begin{gathered}
\langle\vec{J}(t)\rangle=\epsilon j_{d}+(1-\epsilon) j_{n} \\
\langle\bar{D}(t)\rangle=\Phi\left[\epsilon j_{d}+(1-\epsilon) j_{n}\right] t
\end{gathered}
$$

$t>\alpha:$

$$
\begin{gathered}
\langle\bar{J}(t)\rangle=(1-\epsilon) j_{n}+\frac{\epsilon j_{s}}{\pi r^{2}} A(t-\alpha) \\
\langle\bar{D}(t)\rangle=\Phi(1-\epsilon) j_{n} t+\epsilon T+\frac{\epsilon}{\pi r^{2}} V(t-\alpha) .
\end{gathered}
$$

The results of Eq. 21 using the model parameters for Run 1 are plotted parametrically in Figure 7 (curve LS). As expected, $\langle\bar{J}(t)\rangle$ is constant until all of the defects are removed at time $t=\alpha$. The current then increases due to the growth of the pits. Clearly a system of layer-spanning defects cannot produce the current minimums observed in Runs 1-3 of Figure 4. A situation where the depths were constant but not layer-spanning would give results similar to Eq. 21.

Of greater interest, though, is the slope of the line over the course of the etch, defined mathematically as

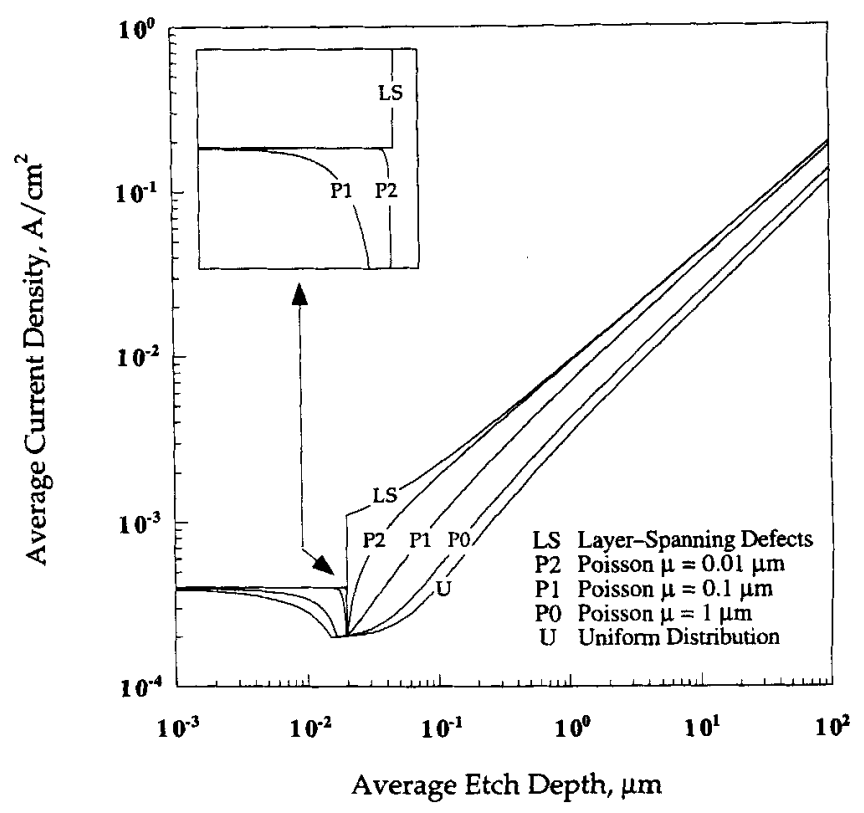

Figure 7. Expected average current density and etch depth, plotted parametrically, for samples with uniformly distributed (U), Poisson distributed with $\mu=0.01 \mu \mathrm{m}, 0.1 \mu \mathrm{m}, 1 \mu \mathrm{m}$ (P), and layer-spanning (LS) defect depths.

The radius and etch rates used in this figure are those from Run 1 in Table 1 . The surface coverage, $\epsilon$, was $0.5 \%$ in all cases.

$$
m \equiv \frac{\partial \log J}{\partial \log D}=\frac{D}{J} \frac{\partial J / \partial t}{\partial D / \partial t} .
$$

For layer-spanning defects, the slope goes to $2 / 3$ monotonically as $\langle\bar{D}(t)\rangle$ (and therefore $t$ ) goes to infinity. This is shown by substituting $\langle\bar{J}(t)\rangle$ and $\langle\bar{D}(t)\rangle$ for $t>\alpha$ from Eq. 21 into Eq. 22 and then taking the limit as $t$ goes to infinity. Physically, a $2 / 3$ slope arises from the geometry of a single pit, whose area, and therefore current contribution, increases with $t^{2}$. Because all of the pits form at the same time, there is no distribution of pit sizes. The slopes for Runs 1,2 , and 3 in Figure 4 are $0.68,0.68$, and 0.72 . However, the data for Run 1 clearly show that the slope passes through a maximum before attaining its steady $2 / 3$ value, which is not possible for a system of layer-spanning defects.

Uniformly Distributed Defect Depths. When defects are rare, the distribution of defect depths approaches a uniform distribution; $f_{x}$ is identically 1 for $0 \leq x \leq 1$ and 0 otherwise. This case is also shown on Figure 7 (curve U). The principal difference between this case and the previous one is that the uniform distribution produces a slope that is not monotonic. The limiting value for $m$ is also a little higher, being 3/4 rather than the $2 / 3$ obtained for a system of layer-spanning defects. This value was computed by evaluating Eqs. 19, 20 and 22 using $f_{x}$ for a uniform distribution. The result was obtained analytically using Maple $\mathrm{V}$, a program for symbolic computational mathematics.

A limiting slope of $3 / 4$ means that the current at long times is increasing with $t^{3}$. This difference in slope is a direct consequence of the distribution of pit sizes resulting from the 
distribution of defect depths. It must be reiterated that these are the limiting slopes expected for very large samples where the variances have become negligible; exact predictions for a finite sample are not possible.

Poisson Distributed Defect Depths. The effects of Poisson distributed defect depths are very similar to those of the uniform distribution, as shown in Figure 7 (curves P0, P1, and P2 corresponding to $\mu=1,0.1$, and 0.01 , respectively). These calculations were also performed analytically using Maple $\mathrm{V}$. The primary distinction is that the slope tends to $2 / 3$ at long times. The maximum in the slope begins to disappear around $\mu=0.05 T$ and is completely gone by $\mu=0.005 T$.

\section{Conclusions}

Noncrystallographic defects in $\mathrm{Al}_{0.4} \mathrm{Ga}_{0.6} \mathrm{As}$ can be revealed by photodissolution using 730-nm (subbandgap) light in nitric acid under closed-circuit conditions. The defects appear as circular channels, indicating a faster etch rate than the surrounding material. Furthermore, the dissolution rate of the defects was found to be dependent on the intensity of the illumination. Since the photon energy of the illumination was below the bandgap energy for $\mathrm{Al}_{0.4} \mathrm{Ga}_{0.6} \mathrm{As}$, the defects must either have a lower band gap than the surrounding material or have a higher concentration of midgap states.

The defects are etch-rate heterogeneities that lead to variations in the overall current as the etch progresses. The progress of the etch can be modeled by formulating model heterogeneities that capture the essential characteristics of the real heterogeneities. In this case, the defects were modeled with a cylindrical geometry and a distribution of defect depths. The distribution was derived assuming that the defects were incorporated during the growth of the epilayer, which produced a uniform distribution under the assertion that defects are rare events. At long times, the overall current for a system of noninteracting defects will grow as $t^{2}$ to $t^{3}$ if the defects are Poisson or uniformly distributed. Other distributions will produce different current-time relationships.

\section{Notation}

$\mathbf{F}=$ Faraday's constant, $\mathrm{C} / \mathrm{eq}$

$h \nu=$ photon energy, eV

$h_{t}=$ heat-transfer coefficient, $\mathrm{mW} / \mathrm{cm}^{2} \cdot{ }^{\circ} \mathrm{C}$

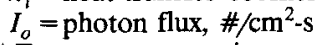

$\Delta T=$ temperature rise at sample surface, ${ }^{\circ} \mathrm{C}$

$(t)=$ total pit area at time $t, \mathrm{~cm}^{2}$

$\mu=$ mean distance between the substrate and beginning of the defect, $\mathrm{cm}$

\section{Literature Cited}

Adachi, S., and K. Oe, "Chemical Etching of GaAs,"J. Electrochem. Soc., 131, 126 (1984).

Badawy, W. A., G. Pfuhl, and W. J. Plieth, "Electrochemical and Photoelectrochemical Behavior of $n-\mathrm{GaAs}$ and $p$-GaAs in the Presence of H2O2," J. Electrochem. Soc., 137, 531 (1990).

Curmmings, K. D., L. R. Harriott, G. C. Chi, and F. W. Ostermayer, Jr., "Using Focused Ion Beam Damage Patterns to Photochemically Etch Features in III-V Materials," Appl. Phys. Lett., 48, 659 (1986).

Fink, Th., and R. M. Osgood, Jr., "Light-Induced Selective Etching of GaAs in AlGaAs/GaAs Heterostructures," J. Electrochem. Soc., 140, L73 (1993).

Hubbard, T. J., and E. K. Antonsson, "Emergent Faces in Crystal Etching," J. Microelectromech. Syst., 3, 19 (1994).

Iwasaki, K., M. Imai, and A. Nakamura, "Round-Robin Test of EPD Measurements on Undoped GaAs Wafers," J. Cryst. Growth, 103, 257 (1990).

Khare, R., and E. Hu, "Resolution Limitations in Focused Ion Beam Damage-Induced Masking for Photoelectrochemical Etching of GaAs," J. Appl. Phys., 72, 1543 (1992).

Khare, R., D. B. Young, and E. L. Hu, "The Bandgap-Selective Photoelectrochemical Etching of $\mathrm{GaAs} / \mathrm{Al}_{x} \mathrm{Gal}_{x}$ As Heterostructures with Varying Mole Fraction,"J. Electrochem. Soc., 140, L117 (1993).

Kuhn-Kuhnenfeld, F., "Selective Photoetching of Gallium Arsenide," J. Electrochem. Soc., 119, 1063 (1978).

Levine, B. F., R. A. Logan, W. T. Tsang, C. G. Bethea, and F. R. Meritt, "Optically Integrated Coherently Coupled $\mathrm{Al}(\mathrm{x}) \mathrm{Ga}(1-\mathrm{x}) \mathrm{As}$ Lasers," Appl. Phys. Lett., 42, 339 (1983).

Mahajan, S., Advances in Electronic Materials, Chap. 10, American Society for Metals, Metals Park, OH, p. 233 (1984).

Malag, A., J. Ratajczak, and J. Gazecki, "Al(x)Ga(1-x)As/GaAs Heterostructure Characterization by Wet Chemical Etching," Mat. Sci. Eng., B20, 332 (1993).

Mao, B. Y., J. A. Nielsen, R. A. Friedman, and G. Y. Lee, "The Applications of Citric Acid/Hydrogen Peroxide Etching Solutions in the Processing of Pseudomorphic MODFETs," J. Electrochem. Soc., 141, 1082 (1994).

Mil'vidskii, M. G., Semiconductor Heterostructures: Physical Processes and Applications, Chap. 5, MIR Publishers, Moscow, p. 76 (1989).

Owen, J. M., and R. H. Rogers, Flow and Heat Transfer in Rotating Disc Systems, Wiley, New York (1989).

Roberston, E. A. III, and H. S. Fogler, "Laser-Enhanced Material Selectivity in N-GaAs/N-Al(0.4)Ga(0.6)As Heterostructures," $J$. Electrochem. Soc., 141, 2893 (1994).

Ruberto, M. N., X. Zhang, R. Scarmozzino, A. E. Willner, D. V. Podlesnik, and R. M. Osgood, "The Laser-Controlled Micrometer-scale Photoelectrochemical Etching of III-V Semiconductors," J. Electrochem. Soc., 138, 1174 (1991).

Stirland, D. J., and B. W. Straughan, "A Review of Etching and Defect Characteristics of Gallium Arsenide Substrate Material," Thin Solid Films, 31, 139 (1976).

Stirland, D. J., G. J. Rees, and A. Ritson, "The Relationship Between Etch Pit Density and Dislocation Density for (001) GaAs," J. Cryst. Growth, 79, 493 (1986).

Manuscript received Sept. 19, 1995, and revision received Dec. 4, 1995. 